\section{ASSISTÊNCIA PRÉ-NATAL E POLÍTICAS PÚBLICAS DE SAÚDE DA MULHER: REVISÃO INTEGRATIVA}

\author{
Prenatal care and public policies for women's health: \\ Integrative review
}

\author{
Atención prenatal y las políticas públicas de la salud de la \\ mujer: revisión integrativa
}

Artigo de Revisão

\section{RESUMO}

Objetivo: Analisar evidências sobre a assistência pré-natal implementada na atenção básica, com foco na percepção de gestantes, puérperas e profissionais de saúde e na relação com as políticas públicas da saúde da mulher. Métodos: Realizou-se um levantamento nas bases de dados BDENF, LILACS e SciELO no período de 2004 a 2013, através dos descritores "assistência pré-natal", "atenção básica", "políticas públicas de saúde" e "assistência integral a saúde da mulher", observando-se publicações em português e espanhol. Selecionaram-se 19 artigos, cujos dados organizados puderam ser analisados à luz das políticas públicas. Resultados: Observou-se que a assistência pré-natal eficaz favorece a diminuição dos índices de mortalidade materna. A percepção de gestantes e puérperas quanto à assistência pré-natal foi discutida com base nas recomendações do Ministério da Saúde e está vinculada à importância da atuação de equipe multiprofissional. Identificaram-se deficiências quanto ao conhecimento das gestantes acerca da atenção odontológica, orientações sobre o aleitamento materno e participação de pais em grupos de educação realizados no pré-natal. Os enfermeiros associaram a realização do pré-natal aos efeitos benéficos, desde que as gestantes realizem as consultas e que os profissionais estejam aptos a adotarem condutas de acompanhamento eficaz no pré-natal. Conclusão: A assistência pré-natal de qualidade garante o acompanhamento da gestante e previne complicações no parto e puerpério, refletindo na diminuição da mortalidade materna e infantil.

Descritores: Assistência pré-natal; Atenção básica; Políticas públicas de saúde; Assistência integral à saúde da mulher.

\section{ABSTRACT}

Objective: To analyze evidence on prenatal care implemented in primary care, with focus on the perception of pregnant women, postpartum mothers and health professionals, and on the relationship with the public policies for women's health. Methods: A survey was conducted in BDENF, LILACS and SciELO databases comprising the period of 2004 to 2013, through the descriptors "Prenatal care", "Primary healthcare", "Public policies for health", "Comprehensive assistance to women's health", and including publications in Spanish and Portuguese language. Nineteen articles were selected, and their data was organized and analysed in light of the public policies. Results: It was observed that effective prenatal care favours the reduction in maternal mortality rates. The perception of pregnant and postpartum women about the prenatal care was discussed on the basis of the Ministry of Health guidelines, and is linked to the importance of the multidisciplinary team's performance. Deficiencies were identified in the women's knowledge with respect to dental care, breastfeeding guidance, and the participation of parents in education groups performed in the prenatal period. Nurses associated the prenatal assistance to beneficial effects, provided that pregnant women perform consultations and professionals are able to adopt effective monitoring in prenatal care. Conclusion: Quality prenatal care ensures the monitoring of pregnant women and prevents complications in childbirth and postpartum period, reflecting on the decrease in maternal and infant mortality.

Descriptors: Prenatal care; Primary care; Public health policies; Comprehensive Health Care to women's health.

\author{
Herla Maria Furtado Jorge ${ }^{(1)}$ \\ Maiza Claudia Vilela Hipólito(2) \\ Valéria Aparecida Masson ${ }^{(2)}$ \\ Raimunda Magalhães da Silva ${ }^{(1)}$
}

1) Universidade de Fortaleza - UNIFOR Fortaleza (CE) Brasil

2) Universidade Estadual de Campinas UNICAMP - Campinas (SP) Brasil
Recebido em: 26/06/2014 Revisado em: 29/07/2014 Aceito em: 09/12/2014 


\section{RESUMEN}

Objetivo: Analizar las evidencias de la atención prenatal establecida en la atención básica investigando la percepción de embarazadas, puérperas y profesionales de la salud y la relación con las politicas públicas de la salud de la mujer Métodos: Se realizó una búsqueda en las bases de datos BDENF, LILACS y SciELO en el periodo entre 2004 y 2013 a través de los descriptores en portugués "assistência pré-natal", "atenção básica", "politicas públicas de saúde” y "assistência integral a saúde da mulher”, observándose las publicaciones en portugués y español. Fueron elegidos 19 artículos cuyos datos fueron analizados basados en las politicas públicas. Resultados: Se observó que la atención prenatal eficaz contribuye para la disminución de los índices de mortalidad materna. La percepción de las embarazadas y puérperas sobre la atención prenatal fue discutida basada en las recomendaciones del Ministerio de la Salud y está vinculada a la importancia de la actuación del equipo multiprofesional. Se identificaron deficiencias en el conocimiento de las embarazadas sobre la atención odontológica, orientaciones sobre el amamantamiento materno y la participación de padres en grupos de educación realizados durante el prenatal. Los enfermeros asociaron la realización del prenatal con los efectos benéficos una vez que las embarazadas participen de las consultas y que los profesionales estén preparados para la adquisición de conductas de seguimiento eficaz en el prenatal. Conclusión: La atención prenatal de calidad garantiza el seguimiento de la embarazada y previene las complicaciones del parto y puerperio, reflejando en la disminución de la mortalidad materna e infantil.

Descriptores: Atención prenatal; Atención primaria de salud; Politicas públicas de Salud; Atención integral de salud.

\section{INTRODUÇÃO}

A Estratégia Saúde da Família (ESF), implementada pelo Ministério da Saúde (MS), pressupõe ações coletivas que possibilitam a realização de uma assistência à saúde na atenção básica $(\mathrm{AB})$ de forma integral, universal, como foco na equidade. Dentre os programas desenvolvidos nesse contexto, preconizam-se ações direcionadas à saúde da criança, adulto, idoso e, sobretudo, à assistência à saúde da mulher ${ }^{(1,2)}$.

As ações de saúde estabelecidas na assistência à saúde da mulher perpassam a prevenção do câncer de colo uterino, o planejamento familiar, a prevenção dos problemas odontológicos em gestantes e a assistência ao pré-natal ${ }^{(2)}$. Esta última agrega um conjunto de procedimentos e cuidados dos quais fazem parte o pré-natal, o diagnóstico de gravidez, a classificação de risco gestacional desde a primeira consulta, além da suplementação alimentar para gestantes com baixo peso, vacinação antitetânica, avaliação do puerpério, entre outros cuidados ${ }^{(3)}$.
Uma análise histórica sobre as políticas públicas de saúde da mulher no Brasil revela que, até a década de 1970, priorizou-se a saúde materno-infantil. Destacam-se nesse período documentos importantes, como: Diretrizes Gerais da Política Nacional de Saúde Materno-Infantil, que instituía a criação de programas voltados para a assistência ao parto, puerpério e à gravidez de risco; Programa de Saúde Materno-Infantil (PSMI), com foco na relação entre a nutrição infantil, gestantes, puérperas, parturientes e mulheres em idade fértil; e o Programa de Prevenção da Gravidez de Alto Risco, com a finalidade de prevenir as gestações de alto risco(4).

A partir dos anos 1980, as políticas de saúde da mulher perdem o enfoque gravídico, privilegiando sua saúde integral com a criação do Programa de Assistência Integral à Saúde da Mulher (PAISM), visando à inclusão da assistência à mulher desde adolescência até a terceira idade, passando a respeitar e comprometer-se com os seus direitos $^{(5)}$.

Duas décadas se passaram e em 2004 o MS instituiu a Política Nacional de Assistência Integral à Saúde da Mulher (PNAISM), que incorporava um enfoque de gênero, integralidade e promoção da saúde como princípios norteadores, buscando consolidar avanços no campo dos direitos sexuais e reprodutivos, com ênfase na melhoria da atenção obstétrica, no planejamento familiar, na atenção ao abortamento inseguro e no combate à violência doméstica e sexual ${ }^{(6)}$.

Da instituição do PAISM até a implementação do PNAISM, reflexões foram necessárias para que a sociedade compreendesse o conceito da mulher contemporânea, livre, independente e com plenos poderes de exercer suas capacidades nos vários contextos sociais, sob o amparo da Constituição, como o direito inalienável de vivenciar sua sexualidade e optar pela não maternidade ${ }^{(())}$

Dessa forma, vale ressaltar o aumento expressivo no número de mulheres em idade fértil no Brasil nos últimos anos, alcançando a marca de 58.404.409, o que representa $65 \%$ do total da população feminina ${ }^{(7)}$. Isso leva o Sistema Único de Saúde (SUS) a se empenhar no desenvolvimento de políticas públicas que contemplem a atenção prénatal e puerperal qualificada, a fim de facilitar o acesso e o encaminhamento da gestante aos serviços de atenção à saúde obstétrica e neonatal, e, acima de tudo, humanizada, promovendo "o vínculo entre a assistência ambulatorial e o momento do parto"(8).

Com base nesses dados, surgiu o seguinte questionamento: como a assistência pré-natal implementada na atenção básica é caracterizada, na percepção de gestantes, puérperas e profissionais de saúde, ao se considerar as recomendações das políticas públicas da saúde da mulher? Com base nesse questionamento, objetivou-se realizar uma 
revisão integrativa da literatura, com a finalidade de analisar evidências sobre a assistência pré-natal implementada na atenção básica, com foco na percepção de gestantes, puérperas e profissionais de saúde, e a relação com as políticas públicas da saúde da mulher.

\section{MÉTODOS}

Trata-se de um estudo de revisão integrativa da literatura ${ }^{(9)}$, realizado no período de maio a julho de 2012.

A presente revisão integrativa contempla as seguintes etapas: definição da questão norteadora (problema) e objetivos da pesquisa; estabelecimento de critérios de inclusão e exclusão das publicações (seleção da amostra); busca na literatura; análise e categorização dos estudos, apresentação e discussão dos resultados ${ }^{(9)}$.

Realizou-se a busca dos artigos indexados nas bases de dados BDENF, LILACS e SciELO, por meio dos descritores "assistência pré-natal", "atenção básica", "políticas públicas de saúde", e "assistência integral a saúde da mulher", os quais foram integrados por meio do operador lógico booleano "and". Optou-se por bases de dados que contemplam a literatura publicada nos países da América Latina e Caribe, como também referências técnicocientíficas brasileiras publicadas no período de 2004 a 2013, em língua portuguesa e em espanhol. Escolheu-se o referido período de busca porque 2004 foi o ano de publicação do PNAISM e em 2013 os últimos estudos publicados.

Os critérios de inclusão englobaram: pesquisas que abordassem a assistência pré-natal, as políticas públicas de saúde da mulher, bem como a percepção da assistência pré-natal sob o ponto de vista de gestantes, puérperas e profissionais de saúde publicadas em português e em espanhol, em formato de artigos. Excluíram-se os artigos que não apresentaram textos publicados na íntegra.

Para facilitar a seleção, categorização das informações e análise dos estudos, elaborou-se um instrumento composto pelos seguintes itens: autor, ano, local, fenômeno estudado, população estudada e resultado. Os artigos selecionados foram lidos exaustivamente e as informações obtidas foram apresentadas na forma de quadro e analisadas à luz das políticas e programas do MS sobre a assistência prénatal, obtendo-se três temas: perfil da assistência pré-natal, percepção de gestantes e puérperas quanto à assistência prénatal e significados atribuídos por enfermeiros à assistência pré-natal.

\section{RESULTADOS}

A pesquisa em base de dados resultou em 57 artigos na base SciELO, 325 na LILACS e 193 na BDENF. Após leitura dos resumos, selecionaram-se 22 na base SciELO, 76 na LILACS e 53 na BDENF.

Posteriormente, restaram 19 artigos, os quais constituíram a amostra do estudo, considerando os critérios de inclusão estabelecidos (Figura 1) e os artigos contemplando autor, objetivo, população e principais resultados sobre o perfil da assistência pré-natal (Quadro I); percepção de gestantes e puérperas quanto à assistência pré-natal (Quadro II); e percepção e atuação do enfermeiro quanto à assistência pré-natal (Quadro III).

Com relação à metodologia empregada, 15 estudos utilizaram abordagem qualitativa e quatro, quantitativa. Quanto ao local da pesquisa e autoria, observou-se que quatro dos estudos foram conduzidos em São Paulo, cinco no Rio de Janeiro, quatro no Rio Grande do Sul, dois em Pernambuco, um em Natal, um no Ceará e dois em Minas Gerais, todos publicados no período de 2004 a 2013.

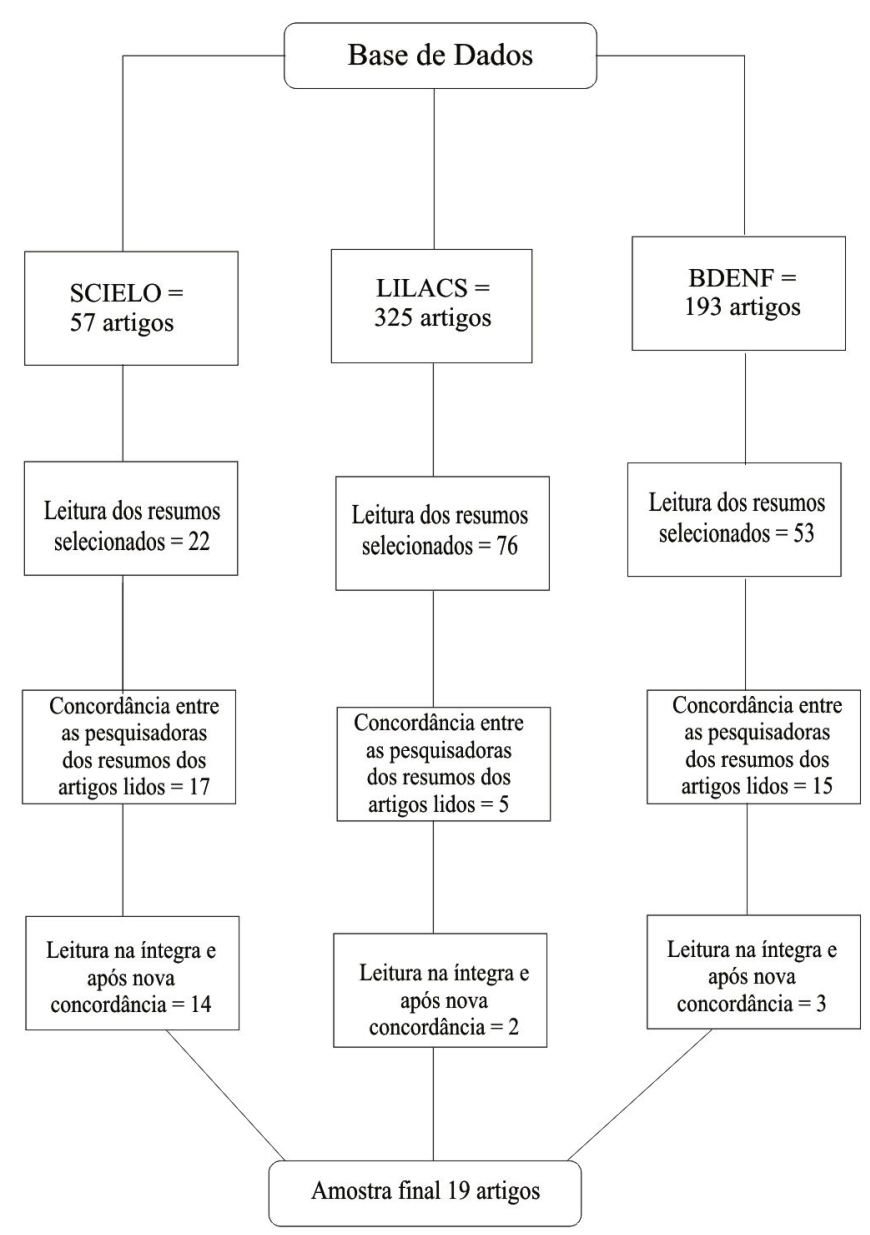

Figura 1 - Fluxograma da descrição da busca dos artigos nas bases de dados. Fortaleza-CE, 2012. 
Quadro I - Descrição dos artigos sobre o perfil da assistência pré-natal, segundo autor, população, objetivo do estudo e principais resultados. Fortaleza-CE, 2012.

\begin{tabular}{|c|c|c|c|}
\hline Autor & População & Objetivo & Principais resultados \\
\hline $\begin{array}{l}\text { Oliveira, } \\
2010^{(10)}\end{array}$ & Neonatos & $\begin{array}{l}\text { Identificar o efeito da gravidez } \\
\text { na adolescência para a } \\
\text { mortalidade fetal. }\end{array}$ & $\begin{array}{l}\text { A gravidez na adolescência surgiu com um efeito } \\
\text { direto para o óbito pós-neonatal. }\end{array}$ \\
\hline $\begin{array}{l}\text { Lago, } \\
2010^{(11)}\end{array}$ & $\begin{array}{l}\text { Gestante e } \\
\text { puérpera }\end{array}$ & $\begin{array}{l}\text { A apresentação prévia de um } \\
\text { fluxograma para estruturação da } \\
\text { linha de cuidado nos sistemas } \\
\text { de saúde. }\end{array}$ & $\begin{array}{l}\text { São apresentados instrumentos de gestão do cuidado } \\
\text { que podem ser utilizados em cada momento do } \\
\text { processo assistencial. }\end{array}$ \\
\hline $\begin{array}{l}\text { Reberte, } \\
2010^{(12)}\end{array}$ & $\begin{array}{l}\text { Oito gestantes e } \\
\text { quatro maridos }\end{array}$ & $\begin{array}{l}\text { Descrever a experiência de pais } \\
\text { em grupo de educação para a } \\
\text { saúde na assistência pré-natal. }\end{array}$ & $\begin{array}{l}\text { A participação do pai nas atividades educativas da } \\
\text { assistência pré-natal produz benefícios para ele e, } \\
\text { consequentemente, para sua família e a sociedade. }\end{array}$ \\
\hline $\begin{array}{l}\text { Morimura, } \\
2006^{(13)}\end{array}$ & 400 puérperas & $\begin{array}{l}\text { Determinar a prevalência de } \\
\text { exame anti-HIV durante o pré- } \\
\text { natal. }\end{array}$ & $\begin{array}{l}\text { Números de consultas realizadas, número de } \\
\text { profissionais que atenderam a mulher no pré-natal, e } \\
\text { a escolaridade associaram-se a realização do exame } \\
\text { anti-HIV. }\end{array}$ \\
\hline $\begin{array}{l}\text { Calderon, } \\
2006^{(14)}\end{array}$ & $\begin{array}{l}\text { Estudo de } \\
\text { revisão }\end{array}$ & $\begin{array}{l}\text { Analisar intervenções efetivas } \\
\text { no pré-natal. }\end{array}$ & $\begin{array}{l}\text { Promoção da saúde materna, prevenção dos riscos e } \\
\text { garantia de suporte nutricional durante a gestação. }\end{array}$ \\
\hline $\begin{array}{l}\text { Minagawa, } \\
2006^{(15)}\end{array}$ & 101 crianças & $\begin{array}{l}\text { Verificar como o peso ao nascer } \\
\text { se relaciona às condições } \\
\text { maternas. }\end{array}$ & $\begin{array}{l}\text { A realização do pré-natal reduziu a ocorrência de } \\
\text { Baixo Peso ao Nascer. }\end{array}$ \\
\hline $\begin{array}{l}\text { Spindola, } \\
2006^{(16)}\end{array}$ & 118 mulheres & $\begin{array}{l}\text { Identificar a clientela traçando } \\
\text { seu perfil epidemiológico }\end{array}$ & $\begin{array}{l}\text { A maioria das mulheres iniciou o pré-natal com } \\
\text { idade gestacional entre } 14 \text { e } 17 \text { semanas, tiveram } \\
\text { anteriormente partos vaginais e não referiram queixas } \\
\text { na primeira consulta }\end{array}$ \\
\hline
\end{tabular}

Quadro II - Descrição dos artigos sobre a percepção de gestantes e puérperas quanto à assistência pré-natal, segundo autor, população, objetivo do estudo e principais resultados. Fortaleza-CE, 2012.

\begin{tabular}{|c|c|c|c|}
\hline Autor & População & Objetivo & $\begin{array}{c}\text { Principais resultados } \\
\end{array}$ \\
\hline Figueiredo, $2008^{(17)}$ & Gestantes & $\begin{array}{l}\text { Analisar o acesso ao pré- } \\
\text { natal na ótica de gestantes } \\
\text { atendidas em Unidade } \\
\text { Básica de Saúde (UBS). }\end{array}$ & $\begin{array}{l}\text { Modificação de alguns aspectos da assistência, } \\
\text { no intuito de adequá-las às reais necessidades das } \\
\text { usuárias e fortalecer o vínculo com os profissionais. }\end{array}$ \\
\hline Paula, 2008 ${ }^{(18)}$ & Gestantes & $\begin{array}{l}\text { Analisar a assistência pré- } \\
\text { natal em UBS. }\end{array}$ & $\begin{array}{l}\text { Apenas uma das gestantes estudadas relatou ter } \\
\text { se submetido a todos os exames e vacinação } \\
\text { recomendados pelo Programa de Humanização no } \\
\text { Pré-Natal e Nascimento. }\end{array}$ \\
\hline Bueno, 2011 ${ }^{(19)}$ & 262 gestantes & $\begin{array}{l}\text { Avaliar as dificuldades no } \\
\text { atendimento de gestantes } \\
\text { com diagnóstico de } \\
\text { toxoplasmose. }\end{array}$ & $\begin{array}{l}\text { O tempo médio de demora na coleta de sangue para os } \\
\text { testes sorológicos no pré-natal foi de } 113,4 \text { dias. Houve } \\
\text { demora média de } 52,1 \text { dias para o encaminhamento e } \\
160,6 \text { dias para o início do tratamento. }\end{array}$ \\
\hline Vargas, $2010^{(20)}$ & Dez puérperas & $\begin{array}{l}\text { Investigar percepçoes } \\
\text { de puérperas sobre sua } \\
\text { participação em grupos de } \\
\text { gestantes. }\end{array}$ & $\begin{array}{l}\text { Faltam ações planejadas e estruturadas nas UBS para } \\
\text { colocar em prática ações educativas e estratégicas, na } \\
\text { efetivação dos grupos de gestantes. }\end{array}$ \\
\hline Batistella, $2006^{(21)}$ & 200 gestantes & $\begin{array}{l}\text { Identificar o conhecimento } \\
\text { de gestantes sobre o pré- } \\
\text { natal odontológico. }\end{array}$ & $\begin{array}{l}\text { Quanto às orientações recebidas sobre saúde } \\
\text { bucal a maioria das entrevistadas não teve } \\
\text { acesso a informações, portanto não apresentaram } \\
\text { conhecimento. }\end{array}$ \\
\hline Dióz, 2006 ${ }^{(22)}$ & Gestantes & $\begin{array}{l}\text { Conhecer a avaliação da } \\
\text { assistência pré-natal na visão } \\
\text { de gestantes. }\end{array}$ & $\begin{array}{l}\text { Expectativa da gestante em ser atendida de modo que } \\
\text { considerarem-se seus problemas orgânicos e também } \\
\text { aqueles relacionados à sua condição feminina. }\end{array}$ \\
\hline Cavalcanti, 2006 ${ }^{(23)}$ & Puérperas & $\begin{array}{l}\text { Identificar fatores de } \\
\text { satisfação de puérperas } \\
\text { quanto à assistência pré- } \\
\text { natal. }\end{array}$ & $\begin{array}{l}\text { Houve relação direta entre as puérperas que relataram } \\
\text { muita satisfação com as orientações recebidas e a } \\
\text { satisfação com o pré-natal. }\end{array}$ \\
\hline
\end{tabular}


Quadro III - Descrição dos artigos sobre a percepção e atuação do enfermeiro quanto à assistência pré-natal, segundo autor, objetivo do estudo, população e principais resultados. Fortaleza-CE, 2012.

\begin{tabular}{|c|c|c|c|}
\hline Autor & População & Objetivo & Principais resultados \\
\hline Narchi, 2010(24) & 131 enfermeiros & $\begin{array}{l}\text { Analisar o exercício } \\
\text { das competências dos } \\
\text { enfermeiros para a atenção } \\
\text { pré-natal. }\end{array}$ & $\begin{array}{l}\text { Os enfermeiros não exercem as competências } \\
\text { essenciais para a atenção qualificada ao } \\
\text { pré-natal, devido às barreiras pessoais e } \\
\text { institucionais com que se defrontam em seu } \\
\text { trabalho. }\end{array}$ \\
\hline Garcia, 2010 & Enfermeiro obstetra & $\begin{array}{l}\text { Identificar atuação do } \\
\text { enfermeiro em nível } \\
\text { primário de atenção à } \\
\text { saúde da mulher. }\end{array}$ & $\begin{array}{l}\text { A atuação do enfermeiro, realizando consultas } \\
\text { de pré-natal de baixo risco na rede básica de } \\
\text { saúde, tem amparo legal e ético, com real } \\
\text { benefício à clientela. }\end{array}$ \\
\hline Valença, 2010 & 18 enfermeiros & $\begin{array}{l}\text { Compreender as ações do } \\
\text { enfermeiro no pré-natal na } \\
\text { prevenção da depressão } \\
\text { puerperal. }\end{array}$ & $\begin{array}{l}\text { As intervenções de Enfermagem realizadas } \\
\text { no pré-natal podem favorecer o bem-estar } \\
\text { geral da mulher, da criança que vai nascer } \\
\text { e da família, contribuindo na prevenção da } \\
\text { depressão puerperal. }\end{array}$ \\
\hline Nery, $2006^{(27)}$ & 12 enfermeiros & $\begin{array}{l}\text { Identificar as ações } \\
\text { desenvolvidas pelo } \\
\text { enfermeiro durante a } \\
\text { consulta pré-natal. }\end{array}$ & $\begin{array}{l}\text { O enfermeiro visa à saúde do binômio } \\
\text { gestante-bebê, abordando exclusivamente } \\
\text { questões relacionadas ao fato de estar grávida, } \\
\text { em detrimento do ser mulher. }\end{array}$ \\
\hline Duarte, $2006^{(28)}$ & Enfermeiros & $\begin{array}{l}\text { Descrever as ações do } \\
\text { enfermeiro na atenção ao } \\
\text { pré-natal. }\end{array}$ & $\begin{array}{l}\text { Promove interação e estabelecimento de } \\
\text { vínculo com gestantes e adesão ao pré-natal. }\end{array}$ \\
\hline
\end{tabular}

\section{DISCUSSÃO}

\section{Perfil da assistência pré-natal}

De posse dos artigos analisados, evidenciaramse sete estudos que apontaram a caracterização e o perfil da assistência pré-natal, bem como sua relação com a diminuição da mortalidade materna.

No cenário brasileiro, a preocupação com a saúde materno-infantil remonta à década de 1940, com a criação do Departamento Nacional da Criança, que enfatizava não somente cuidados com as crianças, mas também com as mães, no que se referia à gravidez e amamentação ${ }^{(5)}$.

$\mathrm{O}$ perfil da assistência pré-natal tem como princípio fundamental acolher a mulher do início ao fim da gestação, garantindo o nascimento de uma criança saudável e o bemestar materno infantil ${ }^{(29)}$.

O perfil epidemiológico de mulheres atendidas na consulta pré-natal de um hospital universitário foi investigado por meio de 118 fichas de atendimento cadastradas de janeiro a junho de 2003. Constatou-se que a maioria dos óbitos relacionados diretamente com a função reprodutiva ocorreu devido à hipertensão na gravidez, complicações no trabalho de parto, hemorragia e infecção puerperal $^{(16)}$.
Destaca-se o Programa de Humanização no PréNatal e Nascimento no âmbito do SUS, publicado em 2002 pela MS, que a partir de 2001 desenvolveu capacitações para atenção humanizada ao parto/nascimento dedicadas aos profissionais de saúde, instituindo um projeto de especialização de enfermeiras obstétricas ${ }^{(30)}$.

Contudo, evidências apontam que o baixo peso ao nascer não está associado ao trabalho materno nem com o pré-natal, mas com as seguintes variáveis: ganho de peso na gestação inferior a sete quilos, idade menor que 20 anos e ausência de parceiro ${ }^{(31)}$. A variável "ganho de peso ao nascer inferior a sete quilos" elucida a recomendação do MS ao publicar o "Manual do Pré-Natal e Puerpério": atenção qualificada e humanizada, garantindo que a atenção pré-natal seja realizada em conformidade com parâmetros estabelecidos, entre os quais se destacam a avaliação do estado nutricional da gestante e o monitoramento por meio do Sistema de Informação em Vigilância Nutricional (SISVAN); prevenção e tratamento dos distúrbios nutricionais ${ }^{(29)}$.

Diante da ocorrência da mortalidade materna, a assistência pré-natal não pode prever as principais complicações do parto na maioria das mulheres, tais como: hemorragias, septicemias e obstruções do trabalho de parto. 
Porém, intervenções eficazes durante a gravidez poderão favorecer o prognóstico materno ${ }^{(14)}$.

De acordo com o MS, é considerada ideal a realização de no mínimo seis consultas no pré-natal e uma no período pós-parto, considerando que a primeira consulta seja até a $16^{\mathrm{a}}$ semana de gestação e que, na ausência de risco, o prénatal deverá seguir as recomendações para atenção básica na assistência pré-natal ${ }^{(29)}$.

Com base na publicação anterior, ressalta-se a importância da solicitação e realização dos exames essenciais no pré-natal, como a testagem anti-HIV, a qual deve ser realizada no primeiro e terceiro trimestres da gestação. Uma pesquisa demonstrou a prevalência da testagem no prénatal, evidenciando que o número de consultas realizadas no pré-natal, o número de profissionais que atenderam no pré-natal e a escolaridade tiveram associação significativa com a realização do exame anti-HIV ${ }^{(13)}$.

\section{Percepção de gestantes e puérperas quanto à assistência pré-natal}

Neste tema, identificaram-se sete estudos realizados em diversas regiões do país que mencionaram a percepção de gestantes e puérperas quanto à assistência pré-natal e foram discutidos com base nas recomendações do MS. Baseado nessas recomendações, analisaram-se o acesso das gestantes à assistência pré-natal, a percepção de gestantes e puérperas, o conhecimento quanto à atenção odontológica $\mathrm{e}$ orientações sobre-aleitamento materno e a participação de pais em grupos de educação realizados no pré-natal.

Uma pesquisa realizada na cidade de Porto Alegre, com a finalidade de analisar o acesso ao pré-natal na atenção básica (AB) sob a ótica de 14 gestantes atendidas em uma Unidade Básica de Saúde (UBS), evidenciou duas formas de acesso ao atendimento: uma por pronto atendimento médico e a outra por consulta agendada. No entanto, dificuldades de acesso foram destacadas para que a gestante conseguisse esse primeiro contato. Percebeu-se a importância da assistência fornecida pela equipe multiprofissional, a fim de atender ao princípio da integralidade. Contudo, observaramse obstáculos em relação à organização da $\mathrm{AB}$, os quais podem dificultar o atendimento às gestantes ${ }^{(17)}$.

No estado do Rio Grande do Sul, um estudo realizado com oito puérperas objetivou conhecer a percepção destas sobre o atendimento em serviço pré-natal e evidenciou que há necessidade de reorganização da atenção no pré-natal e nascimento, sob a lógica da longitudinalidade do cuidado, tanto nos serviços públicos como privados, e de pactuação de ações intersetoriais nos modos de promoção da saúde das mulheres e de fomento à formulação de políticas públicas mais equânimes e positivas na perspectiva da integralidade da atenção ${ }^{(32)}$.
Quanto às dificuldades encontradas frente ao acesso das gestantes, verificaram-se as relações estabelecidas entre equipe de saúde e usuárias, os nós críticos e os ruídos dialógicos apresentados no processo do pré-natal como número reduzido da efetivação do exame de citologia oncótica e inexistência de atividades educativas. No entanto, verificou-se certa resolubilidade na assistência às gestantes, pois grande parcela destas demonstrou satisfação com as consultas realizadas pelos médicos e enfermeiros ${ }^{(33)}$.

Em Viçosa-MG, 44\% das gestantes haviam realizado três consultas pré-natais ou menos, apenas uma das gestantes relatou realização de todos os exames e vacinação recomendados pelo Programa de Humanização no PréNatal e Nascimento e verificou-se falta de conscientização das gestantes quanto aos seus direitos e possíveis formas de reivindicação para acompanhamento de saúde adequado nessa fase da vida ${ }^{(34)}$.

Quanto à assistência odontológica, na cidade de Santa Maria-RS, evidenciou-se que a maioria das entrevistadas não obteve informações sobre saúde bucal e as gestantes de clínicas particulares fizeram maior uso de vitaminas e obtiveram mais orientações sobre aleitamento materno quando comparadas com as gestantes do SUS, o que ressalta a necessidade de maior enfoque odontológico e fonoaudiológico para as gestantes, a fim de propiciar atenção holística para o binômio mãe-bebê $\hat{e}^{(24)}$.

A coerência que norteia o uso do sistema público pelas usuárias relaciona-se ao bom atendimento e à relação pautada por afetividade com os profissionais de saúde ${ }^{(35,13)}$. $\mathrm{Na}$ percepção de 215 puérperas internadas no setor de internação obstétrica do Hospital Universitário Unidade Materno Infantil (HUUMI), identificou-se que o fator que interfere na satisfação e/ou insatisfação das puérperas foi a incipiência dos serviços de pré-natal para atender de forma eficaz a demanda e, consequentemente, favorecer a migração de usuários de uma unidade para outra, acarretando uma demanda maior do que o previsto ${ }^{(13)}$.

Os dados da literatura mostraram que não há participação ativa dos pais no processo da amamentação e que os serviços de saúde devem estabelecer metas claras, instituindo uma política de capacitação da equipe de saúde como padrão de qualidade da assistência prestada à mulher, à criança e ao pai ${ }^{(12,18)}$.

\section{Significados atribuídos por enfermeiros à assistência pré-natal}

Neste tema, identificaram-se cinco estudos realizados em diversas regiões do país que mencionaram a percepção de enfermeiros quanto à assistência pré-natal.

De acordo com as diretrizes propostas pelo Manual Técnico do Pré-Natal e Puerpério, a atenção pré-natal e 
puerperal de qualidade e humanizada é composta por um conjunto de condutas acolhedoras e sem intervenções desnecessárias, de fácil acesso aos serviços de saúde de qualidade, com ações em todos os níveis de atenção promoção, prevenção e assistência à saúde da gestante e do recém-nascido -, indo do atendimento ambulatorial básico ao atendimento hospitalar para alto risco ${ }^{(3)}$.

Frente a essas recomendações, buscou-se analisar como a assistência pré-natal está sendo caracterizada na percepção dos enfermeiros por meio dos resultados de estudos que abordaram: a atuação do enfermeiro na consulta de enfermagem no pré-natal, a atuação da enfermagem obstétrica no pré-natal de baixo risco e a atenção prénatal ${ }^{(31,27)}$

Um estudo sinaliza o interesse da clientela em adquirir novos conhecimentos e a realização de consultas para o acompanhamento da gestação, bem como para as possibilidades e limites de implementação da melhoria da assistência prestada ${ }^{(32)}$

O profissional enfermeiro busca contribuir para uma gravidez sem complicações, prazerosa, e um nascimento saudável, com a atuação direcionada à saúde do binômio gestante-bebê $\hat{(}^{(27)}$. Ressaltam-se os efeitos benéficos para a saúde das gestantes ao constatarem que estas estão interessadas em promover sua qualidade de vida, cumprindo com a realização de consultas, e que os profissionais estão aptos e disponíveis a adotarem condutas benéficas para a saúde das gestantes ${ }^{(32,27)}$.

Vale ressaltar que tais ações vão ao encontro do que é preconizado pelo Manual Técnico de Assistência Pré-natal do Ministério da Saúde, ao estabelecer que as mulheres que têm acesso ao serviço de saúde devem ser informadas sobre programas, palestras e atividades, assim como orientação quanto ao exercício da sexualidade, fisiologia da reprodução, regulação da fertilidade e riscos do aborto provocado, prevenção de doenças sexualmente transmissíveis, câncer cérvico uterino e de mama, e melhoria dos hábitos higiênicos e dietéticos ${ }^{(3)}$.

Destacam-se os resultados alcançados pelo projeto de extensão universitária "Enfermagem obstétrica no atendimento pré-natal de baixo risco", que enfatizou a relação com as políticas públicas de saúde e educação e suas repercussões na Faculdade de Enfermagem/UERJ, constatando que os docentes conseguiram espaço apropriado para promover ações de ensino/assistência, conforme a realidade social das clientes e o hábito profissional dos enfermeiros obstétricos, e implementou as diretrizes do Programa de Assistência Integral à Saúde da Mulher ${ }^{(25)}$.

Certamente a atuação do enfermeiro obstétrico na assistência pré-natal dispõe de uma assistência de qualidade com garantia dos direitos da mulher, pois se sabe que, atualmente, a consulta de enfermagem na rede básica de saúde é realizada de acordo com o roteiro estabelecido pelo MS, garantida pela Lei do Exercício Profissional e o Decreto no 94.406/87. O pré-natal de baixo risco pode ser inteiramente acompanhado pelo enfermeiro ${ }^{(3)}$.

\section{CONCLUSÃO}

As evidências científicas acerca da assistência prénatal implementada na atenção básica apontam para uma associação com as políticas públicas de saúde da mulher. Com relação ao perfil da assistência pré-natal, constatouse que o acompanhamento específico da gravidez garante a qualidade da assistência ao parto e puerpério e a diminuição da mortalidade materno e infantil. A literatura sinaliza que a maioria dos problemas da gravidez pode ser prevenida, tais como: hipertensão gestacional, complicações no trabalho de parto, hemorragia e infecção puerperal.

A percepção de gestantes e puérperas sobre a assistência pré-natal no Brasil relacionou-se ao acesso à assistência pré-natal por meio do pronto atendimento médico ou por consulta agendada; a orientação incipiente sobre a assistência odontológica; a importância do aleitamento materno e a pouca participação dos pais em grupos de educação em saúde.

O significado atribuído à assistência pré-natal pelos enfermeiros condiz com as ações propostas pelas políticas e programas do MS, que se reflete no desenvolvimento de boas práticas na assistência ao pré-natal, favorecendo uma gravidez sem complicações e um nascimento saudável. Os enfermeiros buscam a promoção da qualidade de vida das gestantes orientando-as sobre a sexualidade, fisiologia da reprodução, regulação da fertilidade, doenças sexualmente transmissíveis, bons hábitos higiênicos e dietéticos.

\section{REFERÊNCIAS}

1. Starfield B. Atenção primária: equilíbrio entre necessidades de saúde, serviços e tecnologia. Brasília: UNESCO; 2004.

2. Ministério da Saúde (BR). Alma-ata - Conferência internacional sobre cuidados primários de saúde. Brasília: Ministério da Saúde; 2001.

3. Ministério da Saúde (BR). Assistência pré-natal: manual técnico. Brasília: Ministério da Saúde; 2000.

4. Ministério da Saúde (BR), Secretaria Nacional de Saúde, Coordenação de Proteção MaternoInfantil. Programa nacional de saúde materno-infantil. Brasília: Ministério da Saúde; 1975.

5. Nagahama EEI, Santiago SM. O cuidado pré-natal em hospital universitário: uma avaliação de processo. Cad Saúde Pública. 2006;22(1):173-9. 
6. Ministério da Saúde (BR). Política nacional de atenção integral à saúde da mulher: princípios e diretrizes. Brasília: Ministério da Saúde; 2004.

7. Ministério da Saúde (BR). Política nacional de atenção integral à saúde da mulher: princípios e diretrizes. Brasília: Ministério da Saúde; 2011. [acesso em 2015 Ago 06]. Disponível em: http://bvsms.saude.gov.br/ bvs/publicacoes/politica_nacional_mulher_principios_ diretrizes.pdf

8. Serruya SJ, Cecatti JG, Lago TG. Programa de humanização no pré-natal e nascimento do ministério da saúde no Brasil: resultados iniciais. Cad Saúde Pública. 2004;20(5):1281-9.

9. Mendes KDS, Silveira RCCP, Galvão CM. Revisão integrativa: método de pesquisa para a incorporação de evidências na saúde e na enfermagem. Texto \& Contexto Enferm. 2008;17(4):758-64.

10. Oliveira EFV, Gama SGN, Silva CMFP. Gravidez na adolescência e outros fatores de risco para mortalidade fetal e infantil no Município do Rio de Janeiro, Brasil. Cad Saúde Pública. 2010; 26(3):567-78.

11. Secretaria da Saúde do Estado de São Paulo (BR). Atenção à gestante e à puérpera no SUS-SP: linha de cuidado da gestante e da puérpera. São Paulo: SES/SP; 2010 .

12. Reberte LM, Hoga LAK. A experiência de pais participantes de um grupo de educação para saúde no pré-natal. Cienc Enferm. 2010;16(1):105-14.

13. Morimura MCR, Souza AI, Alencar LCA. Testagem anti-HIV em parturientes admitidas em uma maternidade-escola na cidade do Recife: um estudo transversal. An Fac Med Univ Fed Pernamb. 2006;51(1):4-11.

14. Calderon IMP, Cecatti JG, Veja CEP. Intervenções benéficas no pré-natal para prevenção da mortalidade materna. Rev Bras Ginecol Obstet. 2006;28(5):310-15.

15. Minagawa AT, et al. Baixo peso ao nascer e condições maternas no pré-natal. Rev Esc Enferm USP. 2006;40(4):548-54.

16. Spindola T, Penna LHG, Progianti JM. Perfil epidemiológico de mulheres atendidas na consulta do pré-natal de um hospital universitário. Rev Esc Enferm USP. 2006;40(3):381-8

17. Figueiredo PP, Rossoni E. O acesso à assistência prénatal na atenção básica à saúde sob a ótica das gestantes. Rev Gaúch Enferm. 2008;29(2):238-45.
18. Paula AO, Sartori AL, Martins CA. Aleitamento materno: orientações, conhecimento e participação do pai nesse processo. Rev Eletrônica Enferm [periódico na Internet]. 2010 [acesso em 2014 Fev 03]; 12(3):46470. Disponível em: http://www.fen.ufg.br/revista/v12/ $\mathrm{n} 3 / \mathrm{v} 12 \mathrm{n} 3 \mathrm{a} 07 . \mathrm{htm}$

19. Bueno WF. Difficulties observed in a reference center in the diagnosis and management of pregnant women with toxoplasmosis. Sci Med. 2010;20(1):40-4.

20. Vargas L, Zuse CL. Percepções de puérperas e as experiências em relação ao grupo de gestante. Nursing (São Paulo). 2010:14(149):524-8.

21. Batistella FID, Imparato JCP, Raggio DP, Carvalho AS. Conhecimento das gestantes sobre saúde bucal: na rede pública e em consultórios particulares. RGO (Porto Alegre). 2006;54(1):67-73.

22. Dióz M. Percepção de mulheres grávidas acerca da assistência pré-natal. REME Rev Min Enferm. 2006;10(8):369-37.

23. Cavalcanti LR, Rios CTF, Mochel EG. Satisfação de puérperas com o pré-natal. Nursing (São Paulo). 2006;9(101):1070-4.

24. Narchi NZ. Atenção pré-natal por enfermeiros na Zona Leste da cidade de São Paulo - Brasil. Rev Esc Enferm USP. 2010;44(2):266-73.

25. Garcia SAL, Lippi UG. A necessidade de inserção do enfermeiro obstetra na realização de consultas de pré-natal na rede pública. Einstein (São Paulo). 2010;8(2):241-7.

26. Valença CN, Germano RM. Prevenindo a depressão puerperal na Estratégia Saúde da Família: ações do enfermeiro no pré-natal. Rev RENE. 2010;11(2):12939.

27. Nery TA, Tocantins FR. O enfermeiro e a consulta prénatal: o significado da ação de assistir a gestante. Rev Enferm UERJ. 2006;14(1):87-92.

28. Duarte SJH, Andrade SMO. Assistência pré-natal no programa saúde da família. Esc Anna Nery Rev Enferm. 2006;10(1):121-5.

29. Ministério da Saúde (BR). Pré-natal e puerpério atenção qualificada e humanizada. Brasília: Ministério da Saúde; 2005.

30. Ministério da Saúde (BR), Secretaria Executiva, Programa de Humanização no Pré-natal e Nascimento. Brasília: Ministério da Saúde; 2002. 
31. Tamami MI, Minéo BRE, Elizabeth F, Moreira OIMV, Araújo APC, Saldaña OLD. Baixo peso ao nascer e condições maternas no pré-natal. Rev Esc Enferm USP. 2006; 40(4):548-54.

32. Cabral FB, Hirt LM, Van Der Sand ICP. Atendimento pré-natal na ótica de puérperas: da medicalização à fragmentação do cuidado. Rev Esc Enferm USP. 2013;47(2):281-7.

33. Silva RM, Costa MS, Matsue RY, Sousa GS, Catrib AMF, Vieira LJES. Cartografia do cuidado na saúde da gestante. Ciênc Saúde Coletiva. 2012;17(3):635-42.

34. Almeida PHA, Caliman SB, Costa SCL, Miranda CA, Andrade BM, Chácara PR, et al. Atenção prénatal em Viçosa-MG: contribuições para discussão de políticas públicas de saúde. Rev Med Minas Gerais. 2008;18(3):167-74.
35. Majoreth D. Percepção de mulheres grávidas acerca da assistência pré-natal. REME Rev Min Enferm. 2006; 10(4):369-73.

\section{Endereço para correspondência}

Herla Maria Furtado Jorge

Universidade de Fortaleza - UNIFOR

Av. Washington Soares, 1321

Bairro: Edson Queiroz

CEP: 60.811-905 - Fortaleza - CE - Brasil

E-mail: herlafurtado@gmail.com 